CARDIOVASCULAR MEDICINE

\title{
Reverse remodelling of systolic left ventricular contraction pattern by long term cardiac resynchronisation therapy: colour Doppler shows resynchronisation
}

\author{
P Schuster, S Faerestrand, O J Ohm
}

Heart 2004;90:1411-1416. doi: 10.1136/hrt.2003.030197

See end of article for authors' affiliations

.....................

Correspondence to: Dr Peter Schuster, Department of Heart Disease, Institute of Medicine, Haukeland University Hospital, $\mathrm{N}-5021$ Bergen, Norway; peter.schuster@ med.uib.no

Accepted 26 February 2004

\begin{abstract}
Objective: To quantify long term effects of cardiac resynchronisation therapy (CRT) by biventricular pacing in patients with heart failure (HF).

Methods: Regional changes in left ventricular (LV) contraction patterns effected by CRT in 19 patients with HF (12 with ischaemia; mean (SD) age 66 (9) years) with bundle branch block were examined by colour Doppler tissue velocity imaging (c-TVI). Time differences during main systolic tissue velocity peak (SYS) were compared in the basal and mid LV interventricular septum and in the corresponding LV free wall segments.

Results: From baseline to long term (9.8 (3.0) months) CRT, ejection fraction increased from 21.8 (5.4)\% to $30.8(7.6) \%$, LV end diastolic diameter decreased from $7.6(0.9) \mathrm{cm}$ to $7.1(0.8) \mathrm{cm}$, and end systolic diameter decreased from $6.4(1.2) \mathrm{cm}$ to $6.0(1.2) \mathrm{cm}(\mathrm{p}<0.05)$. LV peak tissue velocities were unchanged during follow up. At baseline, SYS in LV free wall was typically delayed by an average of $29 \mathrm{~ms}$ in the basal LV site and by $18 \mathrm{~ms}$ in the mid LV site. The regional movements of the LV free wall and interventricular septum were separated by an average of only $14 \mathrm{~ms}$ and $-4 \mathrm{~ms}(\mathrm{p}<0.05)$ at the basal site and by $-21 \mathrm{~ms}$ and $-16 \mathrm{~ms}$ at the mid LV site during short term and long term CRT, respectively. Conclusions: The improved haemodynamic functions observed during CRT may be explained by a significant resynchronisation of the regional LV movement pattern during long term follow up.
\end{abstract}

T he introduction of cardiac resynchronisation therapy (CRT) by biventricular pacing in patients with severe treatment refractory heart failure (HF) and bundle branch block has resulted in improvements in clinical symptoms, left ventricular (LV) function, and oxygen uptake even during long term follow up. ${ }^{1}$ Evidence for resynchronisation of the LV has been obtained by various imaging techniques. A recent echocardiographic study showed a radial endocardial wall motion resynchronisation of the LV by short term CRT and a direct relation with invasive haemodynamic variables. ${ }^{2}$ Colour Doppler tissue velocity imaging (c-TVI) provides better temporal and spatial resolution than other non-invasive techniques such as gated blood pool scintigraphy, which also has been used to show acute resynchronisation by CRT. ${ }^{3}$ Furthermore, c-TVI even reflects myocardial contractility by measuring movement of cardiac tissue, as shown in both animal experiments ${ }^{4}$ and a human study. ${ }^{5}$ Recently, c-TVI has shown an immediate effect of CRT by reducing the extent of myocardium at the LV base that displays delayed longitudinal contraction (that is, contraction in diastole after closure of the aortic valve), as well as a reduction in the number of segments displaying delayed contraction during long term CRT. ${ }^{6}$ Even during short term CRT, LV resynchronisation has recently been shown by comparing c-TVI with the QRS complex. ${ }^{7}$

In our studies, we have been able to show and for the first time quantify significant resynchronisation of the LV by using C-TVI at baseline and during short term CRT in patients with severe HF. ${ }^{8}$ The objective of the present study was to measure and quantify the potential effects of long term CRT on reverse LV remodelling produced by changes in the longitudinal systolic LV movement pattern of regional LV isovolumic contraction (IVC) and the main systolic tissue velocity peak (SYS).

\section{METHODS \\ Patients}

The regional ethics committee approved the study and the patients gave their informed consent. The study group consisted of two women and 17 men with a mean (SD) age of 66 (9) years with ischaemic cardiomyopathy $(\mathrm{n}=12)$, idiopathic dilated cardiomyopathy $(\mathrm{n}=4)$, valvar heart disease $(n=2)$, and adriamycin induced cardiomyopathy $(\mathrm{n}=1)$. Twelve patients were in sinus rhythm and seven patients had chronic atrial fibrillation, but all patients were studied as one group because in a previous study at our centre we obtained similar findings with the c-TVI method for patients in sinus rhythm and for patients with atrial fibrillation. ${ }^{8}$ All patients had severe HF and were optimally treated with diuretics $(\mathrm{n}=19)$, angiotensin converting enzyme inhibitors $(n=17)$, digitalis $(n=12)$, amiodarone $(\mathrm{n}=2)$, and $\beta$ blockers $(\mathrm{n}=13)$ when tolerated. None of them were candidates for cardiac surgery; 12 patients were taking warfarin, three aspirin, and one clopidogrel. The patients received CRT according to the accepted criteria of ejection fraction $(\mathrm{EF})<35 \%$, QRS width $>130 \mathrm{~ms}$, and severe HF in New York Heart Association (NYHA) functional classes III and IV. c-TVI findings were partially used as additional criteria. The patients were studied at baseline before pacemaker implantation and after short term CRT for $1.4(0.8)$ months and long term CRT for 9.8 (3.0) months, with clinical evaluation, ECG, six minute hall walk test (6MHWT), and echocardiography.

Abbreviations: $6 \mathrm{MHWT}$, six minute hall walk test; CRT, cardiac resynchronisation therapy; c-TVI, colour Doppler tissue velocity imaging; $\mathrm{EF}$, ejection fraction; HF, heart failure; IVC, isovolumic contraction; IVS, interventricular septum; LBBB, left bundle branch block; LV, left ventricular; NYHA, New York Heart Association; SYS, main systolic tissue velocity peak 

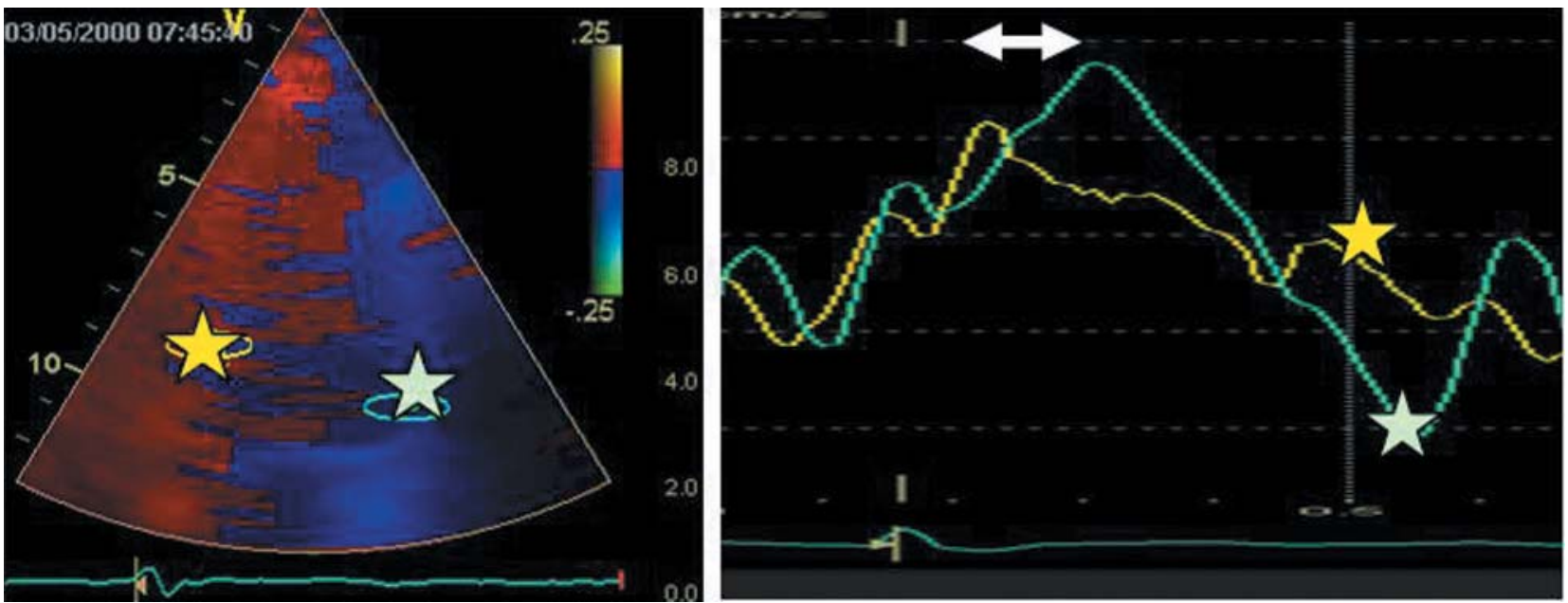

Figure 1 (Left) Colour tissue velocity cine loop in the apical four chamber view (yellow asterisk indicates the interventricular septum; green asterisk indicates the lateral free wall). (Right) Tissue velocity profiles. The arrow shows delayed main systolic peak at the lateral free wall.

\section{Pacemaker system}

The pulse generators implanted were the InSync 8040 $(\mathrm{n}=7)$ and the InSync $8042(\mathrm{n}=12$; Medtronic, Inc, Minneapolis, Minnesota, USA). The InSync 8040 has two ventricular channels connected in parallel, providing similar stimulation of the right and left ventricles. The InSync 8042 has two separate ventricular channels for pacing of the right and left ventricle, respectively, and provides even biventricular pacing as a response to ventricular sensing.

Guided by fluoroscopy, we placed a long guide catheter (Attain LDS model 6216, Medtronic Inc) in the coronary sinus. With the aid of an inflatable balloon catheter, venograms of the coronary veins were made in the frontal and left lateral oblique view. Guided by the venograms, we advanced special coronary vein leads (models $2187(\mathrm{n}=2)$, $4191(\mathrm{n}=3)$, or $4189(\mathrm{n}=14)$; Medtronic Inc) to a stable position in a lateral coronary vein. Acceptable thresholds below $3 \mathrm{~V}$ for LV pacing were obtained. A passive fixation bipolar right ventricular lead was placed in the apex of the right ventricle and a third bipolar lead with passive fixation was placed in the right auricle in patients who were in sinus rhythm $(\mathrm{n}=12)$.

\section{Echocardiographic methods}

An experienced physician (SF) examined the patients in the left lateral recumbent position with a System FiVe digital ultrasound machine (GE Vingmed Ultrasound, Horten, Norway) with a combined phased array transducer providing colour Doppler tissue, two dimensional, M mode, spectral Doppler, and colour Doppler echocardiography. The EF was calculated in accordance with the recommendations of the American Society of Echocardiography with Simpson's rule. ${ }^{9}$ By using M mode echocardiography, the LV end diastolic and end systolic diameters were measured from the parasternal long axis view, also in accordance with American Society of Echocardiography recommendations. During the study, the heart rate was measured from the continuously recorded single channel ECG.

\begin{tabular}{|c|c|c|c|}
\hline & Baseline & Short term follow up & Long term follow up \\
\hline NYHA class & IIII $(3.2(0.6))^{*} \dagger$ & $\|(2.0(0.4))^{*}$ & II $(2.0(0.6)) \dagger$ \\
\hline Sinus rhythm & IIII $(3.1(0.7))^{*} \dagger$ & $\|(2.0(0.5))^{*}$ & $\|(1.8(0.6)) \dagger$ \\
\hline $\mathrm{AF}$ & IIII $(3.3(0.5))^{*} \dagger$ & $\|(2.0(0))^{*}$ & $\|(2.2(0.6)) \dagger$ \\
\hline 6MHWT distance $(\mathrm{m})$ & $400(148)^{*} \dagger$ & $539(121)^{*}$ & $555(163) \dagger$ \\
\hline Ejection fraction (\%) & $21.8(5.4)^{*} \dagger$ & $32.4(8.7)^{*}$ & $30.8(7.6) \dagger$ \\
\hline Sinus rhythm & $22.1(6.0)^{*} \dagger$ & $32.9(10.2)^{*}$ & $30.4(7.7) \dagger$ \\
\hline $\mathrm{AF}$ & $21.4(5.5)^{*}+$ & $31.4(5.6)^{*}$ & $31.4(8.1) \dagger$ \\
\hline LV diastolic volume (ml) & $298(76) \dagger$ & $292(75)$ & $267(67) \dagger$ \\
\hline LV systolic volume (ml) & $224(88)^{*} \dagger$ & $213(82)^{*}$ & $190(78) \dagger$ \\
\hline $\operatorname{LVEDD}(\mathrm{cm})$ & $7.6(0.9) t$ & $7.3(0.9)$ & $7.1(0.8) \dagger$ \\
\hline LVESD $(\mathrm{cm})$ & $6.4(1.2) \dagger$ & $6.3(1.2)$ & $6.0(1.2) \dagger$ \\
\hline IVSdd (cm) & $0.9(0.3)$ & $1.0(0.3)$ & $1.0(0.2)$ \\
\hline IVSds (cm) & $1.3(0.6)$ & $1.2(0.4)$ & $1.4(0.4)$ \\
\hline LVFWdd $(\mathrm{cm})$ & $1.0(0.2)$ & $1.1(0.3)$ & $1.0(0.2)$ \\
\hline LVFWds $(\mathrm{cm})$ & $1.2(0.3)$ & $1.5(0.5) \ddagger$ & $1.2(0.4) \ddagger$ \\
\hline Mitral regurgitation grade & III $(2.9(0.9))^{*} \dagger$ & II $(1.9(1.2))^{*}$ & II (1.8(1.0))† \\
\hline \multicolumn{4}{|c|}{$\begin{array}{l}\text { Data are mean (SD). } \\
\text { * } p<0.05 \text { baseline } v \text { short term follow up; } \uparrow p<0.05 \text { baseline v long term follow up; } ¥ p<0.05 \text { short term v long term } \\
\text { follow up. } \\
6 \text { 6MHWT, } 6 \text { minute hall walk test; AF, atrial fibrillation; IVSdd, interventricular septum diameter during diastole; } \\
\text { IVSds, interventricular septum diameter during systole; LV, left ventricular; LVEDD, left ventricular end diastolic } \\
\text { diameter; LVESD, left ventricular end systolic diameter; LVFWdd, lateral free wall diameter during diastole; } \\
\text { LVFWds, lateral free wall diameter during systole; NYHA, New York Heart Association. }\end{array}$} \\
\hline
\end{tabular}




\section{Colour tissue velocity imaging}

Colour Doppler tissue velocity profiles (fig l) were obtained in the apical four chamber view at frame rates close to 100 frames/s, allowing for accurate evaluation of the LV longitudinal movement pattern at a time resolution of $10 \mathrm{~ms}$ in a post-processing procedure. This procedure has recently been described in detail and has been shown to have acceptable reproducibility of time difference measurement. ${ }^{8}$ The systolic c-TVI peaks appear within $10 \mathrm{~ms}$ in structurally normal hearts. ${ }^{10}$ The simultaneous velocity curves of four selected LV segments were compared for a mean of two consecutive beats. LV tissue velocities and peak tissue velocities were measured during IVC and SYS. The absolute regional time difference between c-TVI peaks in the interventricular septum (IVS) and the LV lateral free wall were measured and compared in basal and mid-LV sites. The four LV segments selected for longitudinal velocity sampling were a basal LV and a mid-LV site in each of the IVS and in the LV free wall.

\section{Statistical analysis}

The data are expressed as mean (SD). The statistical methods used were Student's $t$ test for normally distributed paired and unpaired data and the Mann-Whitney or Kruskal-Wallis test for non-parametric data as appropriate. Commercial software (release 10.1, SPSS Inc, Chicago, Illinois, USA) was used. Significance was set at $\mathrm{p}<0.05$.

\section{RESULTS}

\section{Long term stability of CRT}

The 12 patients in sinus rhythm received an atrial lead for atrioventricular CRT and the seven patients with atrial fibrillation received CRT only. All patients had acceptable thresholds for right atrial and right and left ventricular pacing. We obtained $93 \%$ biventricular pacing at the short term follow up and $96 \%$ biventricular pacing at the long term follow up (not significant). In patients with atrial fibrillation, the average percentage of ventricular pacing was $87 \%$ at short term follow up and $93 \%$ at long term follow up; one patient underwent atrioventricular node ablation. The QRS duration was reduced from $172(30) \mathrm{ms}$ at baseline to 137 (21) ms $(\mathrm{p}<0.05)$ in the course of CRT and was stable at follow up.

\section{Clinical and haemodynamic improvements}

From baseline to both short term and long term follow up, there were significant improvements in NYHA functional class (all patients improved $\geqslant 0.5$ NYHA classes, 15 patients improved $\geqslant 1$ class) and 6MHWT distance (table 1 ). There were no significant differences in these exercise parameters between short term and long term CRT. The EF was significantly improved from baseline to short term CRT and was stable from short term to long term follow up. From baseline to short term follow up, 18 patients improved more than a relative $15 \%$ (average $51.7 \%$ ) in $\mathrm{EF}$; the remaining patient had a stable EF of $30 \%$, although improving two NYHA classes and in 6MHWT from $283 \mathrm{~m}$ to $349 \mathrm{~m}$ from baseline to short term follow up.

An indication of reverse LV remodelling during long term CRT may be the documented significant reduction in both the LV end diastolic and LV end systolic diameters from baseline to long term follow up. The finding of no significant reduction of the LV dimension from baseline to short term CRT indicates that reverse $\mathrm{LV}$ remodelling requires more than 1.4 months on average to take place. However, the LV remodelling had already resulted in a reduction of the systolic LV volume from baseline to short term follow up and in a further reduction to long term follow up. The diastolic volume was first significantly reduced from baseline to long term follow up. M mode IVS and LV free wall dimensions were normal during diastole with no change seen during follow up.

During systole, LV posterior wall thickness was significantly reduced from $1.5(0.5) \mathrm{cm}$ at the short term follow up to $1.2(0.4) \mathrm{cm}(\mathrm{p}<0.05)$ at the long term follow up. Semiquantitatively measured mitral regurgitation was significantly reduced from baseline to short term and long term follow up, respectively. There where no significant differences in any of the echocardiographic parameters between patients in sinus rhythm and patients with atrial fibrillation, except for larger left atria in the atrial fibrillation group during the whole follow up. Clinical improvement was the same in both patients in sinus rhythm and those with atrial fibrillation (table 1). No significant difference in 6MHWT between the groups was observed.

\section{Regional LV contraction pattern during CRT}

During C-TVI imaging, the frame rates were 96 (29) frames/s at baseline, 92 (16) frames/s at short term follow up, and 93 (17) frames/s (not significant) at long term follow up. Heart rates were 75 (20) beats/min at baseline, 82 (11) beats/min at short term follow up, and 79 (15) beats/min (not significant) at long term follow up.

Regional LV mean peak tissue velocity as an expression of regional LV contractility did not change from baseline to either short term or long term CRT (table 2).

A positive LV peak tissue velocity time difference between the IVS and LV free wall in systole indicates that the IVS moved longitudinally towards the apex earlier than the LV free wall. The complete study group had delayed LV free wall movement during both IVC and SYS at the basal and mid-LV sites at baseline. The LV longitudinal movement pattern tended to be more synchronised after short term CRT. During SYS (table 3) basal regional LV contraction was significantly resynchronised, from a 29.1 (47.9) ms delay in the LV free wall at baseline to a 4.2 (58.1) ms delay in the IVS after long term CRT. In the other regions of the LV there was a clear tendency towards resynchronisation from baseline to long

\begin{tabular}{|c|c|c|c|c|}
\hline Site & $\begin{array}{l}\text { Timing of } \\
\text { measurement }\end{array}$ & $\begin{array}{l}\text { Baseline } \\
\text { velocity }(\mathrm{cm} / \mathrm{s})\end{array}$ & $\begin{array}{l}\text { Short term follow } \\
\text { up }(\mathrm{cm} / \mathrm{s})\end{array}$ & $\begin{array}{l}\text { Long term follow } \\
\text { up }(\mathrm{cm} / \mathrm{s})\end{array}$ \\
\hline \multirow[t]{2}{*}{ Basal IVS } & IVC & $2.2(1.9)$ & $1.1(1.8)$ & $1.1(1.5)$ \\
\hline & SYS & $2.1(1.8)$ & $2.5(1.7)$ & $2.0(1.4)$ \\
\hline \multirow[t]{2}{*}{ Mid-IVS } & IVC & $1.9(1.0)$ & $1.6(1.6)$ & $1.3(1.0)$ \\
\hline & SYS & $1.6(2.3)$ & $2.3(2.4)$ & $1.5(1.5)$ \\
\hline \multirow{2}{*}{ Basal LVFW } & IVC & $1.3(1.5)$ & $2.4(1.2)$ & $2.0(1.3)$ \\
\hline & SYS & $2.4(1.0)$ & $2.8(1.6)$ & $2.7(1.6)$ \\
\hline \multirow{2}{*}{ Mid-LVFW } & IVC & $2.6(1.8)$ & $2.7(2.1)$ & $2.3(1.2)$ \\
\hline & SYS & $1.8(1.7)$ & $2.5(2.3)$ & $2.6(1.4)$ \\
\hline
\end{tabular}

Data are mean (SD).

IVC, isovolumic contraction; IVS, interventricular septum; LVFW left ventricular lateral free wall; SYS, main systolic tissue velocity peak. 
Table 3 Main systolic tissue velocity peak time differences at baseline and at follow up for all patients

\begin{tabular}{lllll}
\hline Site & $\begin{array}{l}\text { Timing of } \\
\text { measurement }\end{array}$ & $\begin{array}{l}\text { Baseline time } \\
\text { difference }(\mathrm{ms})\end{array}$ & $\begin{array}{l}\text { Short term } \\
\text { follow up (ms) }\end{array}$ & $\begin{array}{l}\text { Long term } \\
\text { follow up (ms) }\end{array}$ \\
\hline Basal LV & IVC & $19.6(25.9)$ & $12.5(19.1)$ & $9.0(23.3)$ \\
\multirow{3}{*}{ Mid-LV } & SYS & $29.1(47.9)^{*}$ & $13.7(72.7)$ & $-4.2(58.1)^{*}$ \\
& IVC & $27.1(31.1)$ & $9.3(26)$. & $10.9(32.4)$ \\
& SYS & $18.4(61.0)$ & $-20.6(74.0)$ & $-16.2(67.0)$ \\
\hline
\end{tabular}

Data are mean (SD). Positive value: delayed LV free wall; negative value: delayed IVS. ${ }^{*} \mathrm{p}<0.05$.

term CRT during both IVC and SYS but for the study population as a whole, resynchronisation during both short term and long term CRT did not reach significance.

The heterogeneous LV movement pattern in patients with HF with left bundle branch block (LBBB) - delayed LV free wall movement in some patients and a counterintuitive delay in the IVS in basal LV segments in others-was taken into account when the changes in LV movement pattern for the patients with LBBB and delayed LV free wall movement were calculated separately (fig 2). Data for patients with delayed IVS movement were not calculated separately since the number of patients was too small.

In patients with LBBB who displayed a delayed LV free wall at baseline $(n=12$ during IVC at basal and mid LV; $\mathrm{n}=15$ during SYS at basal LV; $\mathrm{n}=12$ during SYS at mid LV) there was a significant change to a more synchronous movement pattern in SYS, which had already occurred at short term follow up. However, in the same patients the LV IVC peak tissue velocities tended towards an almost significant time related further regional LV resynchronisation from short term to long term CRT (fig 2).

Figure 3 shows a c-TVI from a patient with a $65 \mathrm{~ms}$ delay in basal LV free wall movement during SYS compared with the IVS at baseline and significant resynchronisation of LV regional contraction during both short term and long term CRT. In this particular patient the velocity peaks during IVC were better defined after short term and long term CRT than at baseline, which probably contributed to an incremental improvement in LV function in addition to the beneficial effect of a resynchronised SYS.

\section{Limitations}

The number of patients with idiopathic cardiomyopathy was too small to make subgroup analysis meaningful, but the group as a whole represents an average heterogeneous CRT patient population and even the low number of patients is in the range of studies of the time consuming and demanding C-TVI method. ${ }^{711}$ The absence of non-responders in the study may be explained by the fact that the c-TVI examination was a part of the inclusion criteria and therefore is a shortcoming of the study. Non-responders have to be examined in future studies. To measure interventricular asynchrony the scanning sector would have to be increased, which would reduce the frame rate to below 60-70 frames/s. Such low frame rates would prohibit the simultaneous assessment of interventricular asynchrony with current equipment for c-TVI. A promising and more direct measurement of contractility is strain and strain rate based on measurement of tissue velocity gradients between two points within the same segment. ${ }^{12}{ }^{13}$ However, in our experience c-TVI of the enlarged LV has been superior to the even more angle dependent strain/strain rate method to measure the absolute time difference between simultaneously obtained c-TVI profiles. Strain as a means of visualising the temporal aspect of LV contraction had to be abandoned because of the noise and temporal dispersion caused by the large imaging windows, which were necessary to show simultaneously the velocity profiles from both the LV free wall and the IVS independently of QRS. Relative resynchronisation related to the QRS complex ${ }^{7}$ measured by strain needs small echocardiographic sectors and does not allow measurement of the absolute resynchronisation of the simultaneously measured TVI profiles independent of the QRS, which is shown in the present study by c-TVI. For the main objective of examining the pure LV contraction pattern by C-TVI, the atrioventricular interval optimisation was not examined, but the impact on systolic LV contraction pattern was considered to be of minor importance, even if

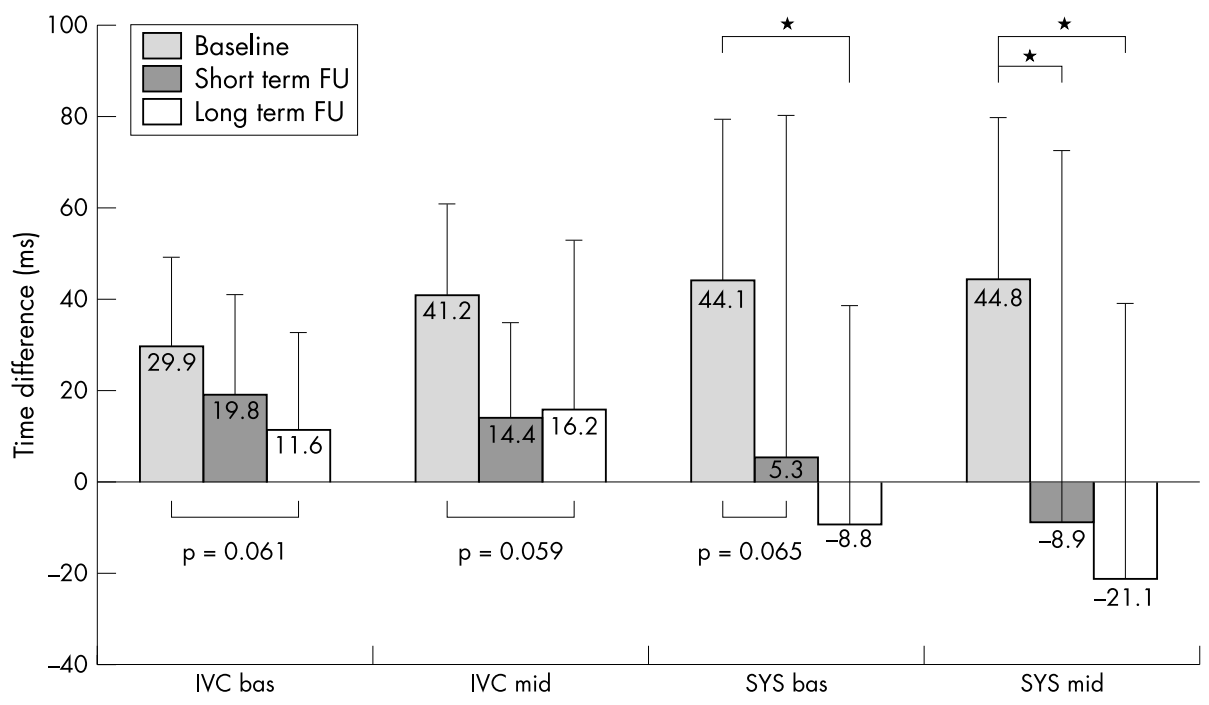

Figure 2 Regional left ventricular (LV) peak tissue velocity time differences during isovolumic contraction (IVC) and main systolic tissue velocity peak (SYS) between the interventricular septum and lateral free wall at the basal (bas) and mid-LV segments in patients with delayed lateral free wall movement at baseline. Time differences are described during baseline and short term and long term follow up (FU). ${ }^{*} \mathrm{p}<0.05$. 

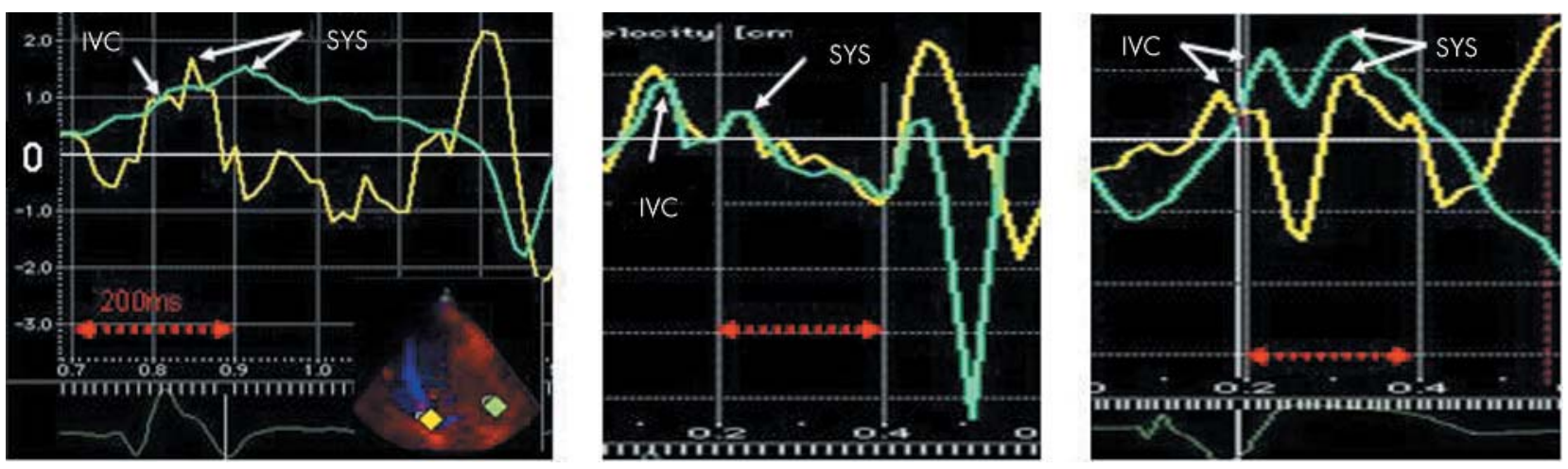

Figure 3 Colour tissue velocity recording of one patient with a $65 \mathrm{~ms}$ delayed basal left ventricular lateral free wall (green line) compared with the interventricular septum (yellow line) during SYS at baseline (left), and with significant resynchronisation after both short term (middle) and long term (right) cardiac resynchronisation therapy. The IVC tissue velocity peak was more clearly defined both at short term and long term follow up than at baseline, which probably contributed to an incremental improvement in left ventricular function, in addition to the beneficial effects of a resynchronised SYS.

haemodynamic and clinical improvements in the study might have been influenced.

\section{DISCUSSION}

\section{Clinical and haemodynamic improvement of HF}

The significant clinical improvements in all patients in NYHA functional class and 6MHWT distance took place from baseline to short term CRT. The significant relative improvement of 51.7 (30.7)\% in EF was documented at short term follow up, suggesting that resynchronisation is important for the acute improvement in LV systolic performance, ${ }^{14}{ }^{15}$ supported by the significant reduction of the systolic LV volumes. The improvement in EF and mitral regurgitation was sustained during long term CRT in accordance with the significant sustained resynchronisation of LV regional contraction during chronic CRT documented in this study. The high percentage of ventricular pacing during follow up as a prerequisite of examining the resynchronising effect of CRT, even in patients with atrial fibrillation (only one patient needing atrioventricular ablation), may be explained by the high percentage of $\beta$ blocker and digitalis use.

\section{Time related reverse remodelling of the $L V$}

The reduction in LV size and volume, indicating reverse remodelling of the severely dilated LV, was significant after at least three months of CRT. This clearly indicates that CRT effects a time related, progressive systolic haemodynamic benefit of resynchronisation. These observations are in accordance with recent studies on the long term effects of CRT. ${ }^{1}$ The impact on the systolic LV free wall dimensions with a reduction from short term to long term follow up may be an indirect sign of time related improved contractility and haemodynamic function.

In addition to gated blood pool scintigraphy with a lower time resolution of $30 \mathrm{~ms}^{16}$ and echocardiographic phase analysis, ${ }^{2}$ c-TVI has shown that short term CRT resynchronises the LV. The c-TVI methods used to show LV resynchronisation by CRT either measure the reduction in delayed longitudinal contraction, ${ }^{17}$ measure a global resynchronisation in a $12 \mathrm{LV}$ segment model correlated to the QRS, ${ }^{7}$ or display absolute resynchronisation of the IVS relative to the LV free wall, as in our study and another study. ${ }^{818}$ The amount of dyssynchrony before CRT between basal IVS and LV free wall movements in our study of $29 \mathrm{~ms}$ is in accordance with the $31 \mathrm{~ms}$ obtained in a study of 25 patients where c-TVI profiles were examined in relation to the $\mathrm{QRS}^{7}$ although in our study we measured the absolute time difference rather than increasing inaccuracy by including the QRS. By using our validated c-TVI method, ${ }^{810}$ this study has shown a further degree of resynchronisation of longitudinal LV contraction during long term CRT. The typical delay in movement of the LV free wall relative to the IVS, during both IVC and SYS at baseline in patients with LBBB, was resynchronised during short term CRT and was further progressively resynchronised during long term CRT. This observation indicates that the improvement is time related, in that long term CRT improves the initial resynchronisation provided by short term CRT, and may explain the long term progressive reverse LV remodelling by CRT.

\section{Colour tissue velocity imaging}

The LV tissue peak velocity time difference of less than $\pm 20 \mathrm{~ms}$ in all four LV sites we examined, during both IVC and SYS, can be regarded as a synchronous contraction based on the time resolution of $10 \mathrm{~ms}$ achieved with this echocardiographic method and on findings in structurally normal hearts. ${ }^{10}$ SYS seems to be more affected by CRT, especially in the patients with a delayed LV free wall at baseline. Our finding that some patients had a counterintuitively delayed IVS, despite LBBB, points in the same direction as recently published studies showing that QRS duration and configuration do not predict the pattern of LV asynchrony in patients with bundle branch block. ${ }^{19} 20$

Regional LV contractility in terms of mean peak tissue velocity during CRT was unchanged in this study. This finding may contribute to a better understanding of the fact that CRT does not increase LV oxygen consumption ${ }^{21}$ and that the improvement in LV systolic function is actually produced by the resynchronising effects of CRT.

\section{Conclusions}

This study clearly shows for the first time the importance of the regional tissue velocity time aspect of LV contraction during long term follow up for the improvement in LV performance obtained by CRT. These findings suggest that the more synchronous contraction pattern of the LV provided by CRT is a major component of improvements in LV function and clinical improvements in HF symptoms.

The changes observed in longitudinal LV resynchronisation exceed previous short term observations with $\mathrm{C}^{-\mathrm{TVI}^{7}}{ }^{8}$ and quantify for the first time the absolute changes in the time aspect of LV contraction during long term follow up. This study helps to improve our understanding of the clinical long term benefits of CRT in patients with HF. However, it is still an open question whether the degree of LV asynchrony 
measured by this c-TVI method in patients with severe HF and bundle branch block before CRT is a predictive parameter for the haemodynamic and clinical improvements produced by CRT. Whether these echocardiographic parameters for asynchronous LV contraction should be regarded as an alternative to the QRS duration, which is normally taken as an indicator of the haemodynamic and clinical effects of CRT, is another open question.

\section{ACKNOWLEDGEMENTS}

This study was supported by the Norwegian Council on Cardiovascular Diseases.

\section{Authors' affiliations}

P Schuster, S Faerestrand, O J Ohm, Department of Heart Disease, Haukeland University Hospital and Institute of Medicine, University of Bergen, Bergen, Norway

\section{REFERENCES}

1 Auricchio A, Stellbrink C, Sack S, et al. Long-term clinical effect of hemodynamically optimized cardiac resynchronization therapy in patients with heart failure and ventricular conduction delay. J Am Coll Cardiol 2002;39:2026-33.

2 Breithardt OA, Stellbrink C, Kramer AP, et al. Echocardiographic quantification of left ventricular asynchrony predicts an acute hemodynamic benefit of cardiac resynchronization therapy. J Am Coll Cardiol 2002;40:536-45

3 Kerwin WF, Botvinick EH, O'Connell JW, et al. Ventricular contraction abnormalities in dilated cardiomyopathy: effect of biventricular pacing to correct interventricular dyssynchrony. J Am Coll Cardiol 2000;35:1221-7.

4 Gorcsan J, Strum DP, Mandarino WA, et al. Quantitative assessment of alterations in regional left ventricular contractility with color-coded tissue Doppler echocardiography: comparison with sonomicrometry and pressurevolume relations. Circulation 1997:95:2423-33.

5 Zamorano J, Wallbridge DR, Ge J, et al. Non-invasive assessment of cardiac physiology by tissue Doppler echocardiography: a comparison with invasive haemodynamics. Eur Heart J 1997;18:330-9.

6 Sogaard P, Egeblad H, Kim WY, et al. Tissue Doppler imaging predicts improved systolic performance and reversed left ventricular remodeling during long-term cardiac resynchronization therapy. J Am Coll Cardiol 2002;40:723-30

7 Yu CM, Chau E, Sanderson JE, et al. Tissue Doppler echocardiographic evidence of reverse remodeling and improved synchronicity by simultaneously delaying regional contraction after biventricular pacing therapy in heart failure. Circulation 2002; 105:438-45.
8 Schuster $\mathrm{P}$, Faerestrand S, Ohm OJ. Colour tissue velocity imaging can show resynchronisation of longitudinal left ventricular contraction pattern by biventricular pacing in patients with severe heart failure. Heart 2003;8:859-64.

9 Schiller NB, Shah PM, Crawford M, et al. Recommendations for quantitation of the left ventricle by two-dimensional echocardiography. American Society of Echocardiography committee on standards, subcommittee on quantitation of two-dimensional echocardiograms. J Am Soc Echocardiogr 1989:2:358-67.

10 Schuster P, Faerestrand S, Ohm OJ, et al. Feasibility of color Doppler tissue velocity imaging for assessment of regional timing of left ventricular longitudinal movement. Scand Cardiovasc J 2004;38:39-45.

11 Breithardt OA, Stellbrink C, Herbots L, et al. Cardiac resynchronization therapy can reverse abnormal myocardial strain distribution in patients with heart failure and left bundle branch block. J Am Coll Cardiol 2003:42:486-94.

12 Abraham TP, Nishimura RA. Myocardial strain: can we finally measure contractility? J Am Coll Cardiol 2001;37:731-4.

13 Edvardsen T, Gerber BL, Garot J, et al. Quantitative assessment of intrinsic regional myocardial deformation by Doppler strain rate echocardiography in humans: validation against three-dimensional tagged magnetic resonance imaging. Circulation 2002;106:50-6.

14 Breithardt OA, Stellbrink C, Franke A, et al. Acute effects of cardiac resynchronization therapy on left ventricular Doppler indices in patients with congestive heart failure. Am Heart J 2002; 143:34-44.

15 Leclercq $\mathrm{C}$, Cazeau S, Le Breton $\mathrm{H}$, et al. Acute hemodynamic effects of biventricular DDD pacing in patients with end-stage heart failure. J Am Coll Cardiol 1998;32:1825-31.

16 Kerwin WF, Botvinick EH, OConnell JW, et al. Ventricular contraction abnormalities in dilated cardiomyopathy: effect of biventricular pacing to correct interventricular dyssynchrony. J Am Coll Cardiol 2000;35:1221-7.

17 Sogaard P, Egeblad H, Pedersen AK, et al. Sequential versus simultaneous biventricular resynchronization for severe heart failure: evaluation by tissue Doppler imaging. Circulation 2002;106:2078-84.

18 Bax JJ, Molhoek SG, van Erven L, et al. Usefulness of myocardial tissue Doppler echocardiography to evaluate left ventricular dyssynchrony before and after biventricular pacing in patients with idiopathic dilated cardiomyopathy. Am J Cardiol 2003;91:94-7.

19 Yu CM, Lin H, Zhang Q, et al. High prevalence of left ventricular systolic and diastolic asynchrony in patients with congestive heart failure and normal QRS duration. Heart 2003;89:54-60.

20 Schuster P, Faerestrand S, Ohm OJ. Color Doppler tissue velocity imaging can disclose systolic left ventricular asynchrony independent of the QRS morphology in patients with severe heart failure. Pacing Clin Electrophysiol 2004;27:460-7.

21 Nelson GS, Berger RD, Fetics BJ, et al. Left ventricular or biventricular pacing improves cardiac function at diminished energy cost in patients with dilated cardiomyopathy and left bundle-branch block. Circulation 2000; 102:3053-9.

\section{FROM BMJ JOURNALS}

\section{Current smoking and the risk of non-fatal myocardial infarction in the WHO MONICA Project populations}

\section{S Mähönen, P McElduff, A J Dobson, K A Kuulasmaa, A E Evans, for the WHO MONICA Project}

Please visit the Heart website [www.heartin com] for a link to the full text of this article.
Background: Cohort studies have shown that smoking has a substantial influence on coronary heart disease mortality in young people. Population based data on non-fatal events have been sparse, however.

Objective: To study the impact of smoking on the risk of non-fatal acute myocardial infarction (MI) in young middle age people.

Methods: From 1985 to 1994 all non-fatal MI events in the age group 35-64 were registered in men and women in the WHO MONICA (multinational monitoring of trends and determinants in cardiovascular disease) project populations ( 18762 events in men and 4047 in women from 32 populations from 21 countries). In the same populations and age groups 65741 men and 66717 women participated in the surveys of risk factors (overall response rate $72 \%$ ). The relative risk of non-fatal MI for current smokers was compared with nonsmokers, by sex and five year age group.

Results: The prevalence of smoking in people aged 35-39 years who experienced non-fatal MI events was $81 \%$ in men and $77 \%$ in women. It declined with increasing age to $45 \%$ in men aged 60-64 years and 36\% in women, respectively. In the 35-39 years age group the relative risk of non-fatal MI for smokers was 4.9 (95\% confidence interval (CI) 3.9 to 6.1 ) in men and 5.3 (95\% CI 3.2 to 8.7 ) in women, and the population attributable fractions were $65 \%$ and $55 \%$, respectively.

Conclusions: During the study period more than half of the non-fatal MIs occurring in young middle age people can be attributed to smoking.

A Tobacco Control 2004;13:244-250 